\title{
Majorana fermions in the Kitaev quantum spin system $\alpha-\mathrm{RuCl}_{3}$
}

\author{
Seung-Hwan Do 1,2, Sang-Youn Park², Junki Yoshitake ${ }^{3}$, Joji Nasu4, Yukitoshi Motome ${ }^{3}$, \\ Yong Seung Kwon ${ }^{5}$, D. T. Adroja ${ }^{6,7}$, D. J. Voneshen ${ }^{6}$, Kyoo Kim ${ }^{2}$, T.-H. Jang ${ }^{2}$, J.-H. Park ${ }^{2,8,9 \star}$, \\ Kwang-Yong Choi ${ }^{1 \star}$ and Sungdae $\mathrm{Ji}^{2,8 \star}$
}

Geometrical constraints to the electronic degrees of freedom within condensed-matter systems often give rise to topological quantum states of matter such as fractional quantum Hall states, topological insulators, and Weyl semimetals $^{1-3}$. In magnetism, theoretical studies predict an entangled magnetic quantum state with topological ordering and fractionalized spin excitations, the quantum spin liquid ${ }^{4}$. In particular, the so-called Kitaev spin model $^{5}$, consisting of a network of spins on a honeycomb lattice, is predicted to host Majorana fermions as its excitations. By means of a combination of specific heat measurements and inelastic neutron scattering experiments, we demonstrate the emergence of Majorana fermions in single crystals of $\alpha-\mathrm{RuCl}_{3}$, an experimental realization of the Kitaev spin lattice. The specific heat data unveils a two-stage release of magnetic entropy that is characteristic of localized and itinerant Majorana fermions. The neutron scattering results corroborate this picture by revealing quasielastic excitations at low energies around the Brillouin zone centre and an hour-glass-like magnetic continuum at high energies. Our results confirm the presence of Majorana fermions in the Kitaev quantum spin liquid and provide an opportunity to build a unified conceptual framework for investigating fractionalized excitations in condensed matter ${ }^{1,6-8}$.

Quantum spin liquids (QSLs) are an unconventional electronic phase of matter characterized by an absence of magnetic longrange order down to zero temperature. They are typically predicted to occur in geometrically frustrated magnets such as triangular, kagome, and pyrochlore lattices ${ }^{4}$, and typically display a macroscopic degeneracy that stabilizes a topologically ordered ground state. The Kitaev QSL state arises as an exact solution of the ideal two-dimensional (2D) honeycomb lattice with bond-directional Ising-type interactions $\left(H=J_{\mathrm{K}}^{\gamma} S_{i}^{\gamma} S_{j}^{\gamma} ; \gamma=x, y, z\right)$ on the three distinct links (Fig. 1a) by expressing the spin excitations in terms of non-interacting Majorana fermions ${ }^{5,9}$. The elementary excitations of a Kitaev QSL are localized and itinerant Majorana fermions ${ }^{5}$, which are associates with static $Z_{2}$-fluxes and propagating quasiparticles (Fig. 1b). These two types of excitation have ramifications for the observable physics and potential technological applications of QSL in quantum computers ${ }^{10-14}$.
As candidates for realizing a QSL, honeycomb iridates $\mathrm{A}_{2} \mathrm{IrO}_{3}$ $(\mathrm{A}=\mathrm{Li}, \mathrm{Na})$ with a spin-orbit coupled $J_{\text {eff }}=1 / 2 \operatorname{Ir}^{4+}\left(5 d^{5}\right)$ state $^{15}$ have been intensively studied. This is due to the orbital state forming the three orthogonal bonds required for the bond-directional exchange interactions in the geometry ${ }^{16}$. The iridates, however, cannot avoid monoclinic distortions with anisotropic Ir-Ir bonds disturbing the exchange frustration away from the ideal values, and their magnetism is apparently dominated by antiferromagnetic (AFM) ordering ${ }^{17,18}$.

A promising candidate for the Kitaev model system is the van der Waals ruthenate $\alpha-\mathrm{RuCl}_{3}$ with $J_{\text {eff }}=1 / 2 \mathrm{Ru}^{3+}\left(4 d^{5}\right)$ ions ${ }^{19,20}$. There is a growing body of evidence that $\alpha-\mathrm{RuCl}_{3}$ hosts predominantly Isinglike Kitaev interactions and that the ground state could be proximate to the QSL state $\mathrm{e}^{21,22}$. Most crystallographic studies reported the presence of the monoclinic distortions ${ }^{23,24}$, resulting in considerable contribution of Heisenberg and asymmetric exchange interactions ${ }^{25,26}$. However, these distortions are probably due to stacking faults of the $\mathrm{RuCl}_{3}$ layers, and even lead to multiple magnetic transitions ${ }^{24}$. Recently, significant advances in the synthesis of high-quality $\alpha-\mathrm{RuCl}_{3}$ crystals have been achieved. These crystals are almost free from stacking faults and have a rhombohedral $(R \overline{3})$ phase, while preserving the Ising-type AFM state below $6.5 \mathrm{~K}$ due to nonvanishing inter-layer couplings ${ }^{27}$. Importantly, this high-symmetry structure renders isotropic Kitaev interactions $\left(J_{\mathrm{K}}=J_{\mathrm{K}}^{x}=J_{\mathrm{K}}^{y}=J_{\mathrm{K}}^{z}\right)$ with a $94^{\circ} \mathrm{Ru}-\mathrm{Cl}-\mathrm{Ru}$ bond angle maximizing the Kitaev interaction, and the Heisenberg contribution becomes minimal ${ }^{26}$. Furthermore, recent methodological progress in the quantum Monte Carlo (QMC) method and cluster dynamic mean-field theory (CDMFT) for thermally excited quantum states provides a route to identify Majorana fermions emerging from the QSL ground state ${ }^{12-14}$. It is predicted that thermally fluctuating quantum spins are successively fractionalized into itinerant and localized Majorana fermions at crossover temperatures $T_{\mathrm{L}}\left(\right.$ low- $T$ ) and $T_{\mathrm{H}}$ (high- $T$ ), respectively. At very low-temperature $\left(T<T_{\mathrm{L}}\right), Z_{2}$-fluxes are mostly frozen in the topologically ordered zero-temperature QSL state and the thermal energy excites only low-energy itinerant Majorana fermions (see Fig. 1b). On increasing the temperature across $T_{\mathrm{L}}$, the fluxes fluctuate to activate localized Majorana fermions (Kitaev paramagnet). Upon further heating, itinerant Majorana fermions are additionally activated and the spin-spin correlation fades out across $T_{\mathrm{H}}$.

\footnotetext{
${ }^{1}$ Department of Physics, Chung-Ang University, Seoul 06974, Republic of Korea. ${ }^{2}$ Max Planck POSTECH/Hsinchu Center for Complex Phase Materials, POSTECH, Pohang 37673, Republic of Korea. ${ }^{3}$ Department of Applied Physics, University of Tokyo, Bunkyo, Tokyo 113-8656, Japan. ${ }^{4}$ Department of Physics, Tokyo Institute of Technology, Meguro, Tokyo 152-8551, Japan. ${ }^{5}$ Department of Emerging Materials Science, DGIST, Daegu 42988, Republic of Korea. ${ }^{6}$ ISIS Facility, Rutherford Appleton Laboratory, Didcot OX11 OQX, UK. ${ }^{7}$ Highly Correlated Matter Research Group, Physics Department, University of Johannesburg, PO Box 524, Auckland Park 2006, South Africa. ${ }^{8}$ Department of Physics, POSTECH, Pohang 37673, Republic of Korea. ${ }^{9}$ Division of Advanced Materials Science, POSTECH, Pohang 37673, Republic of Korea. *e-mail: jhp@postech.ac.kr; kchoi@cau.ac.kr; sungdae@postech.ac.kr
} 


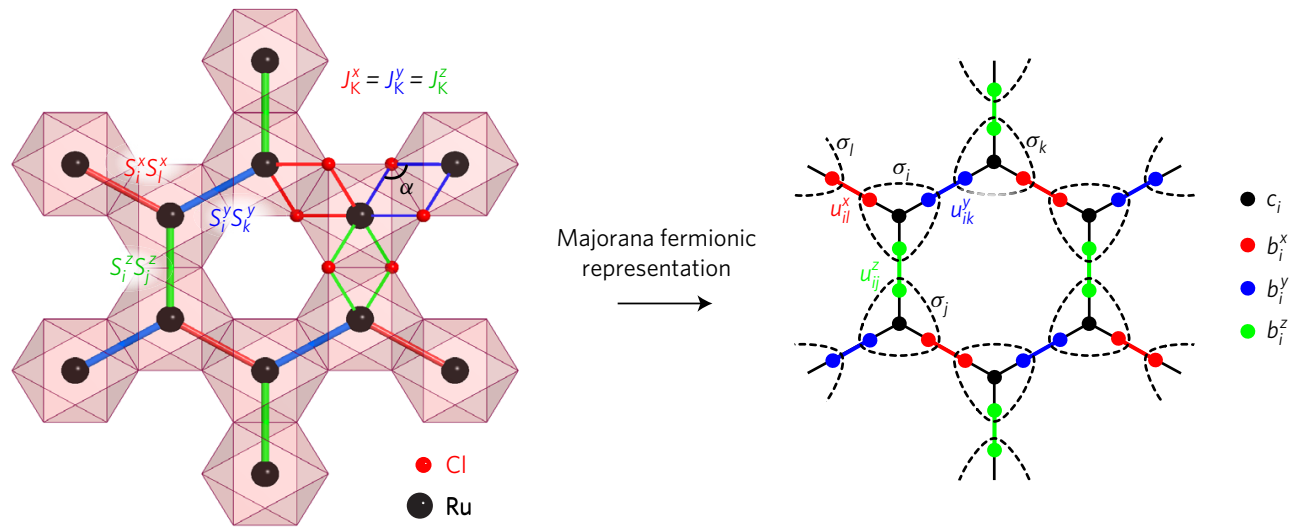

b
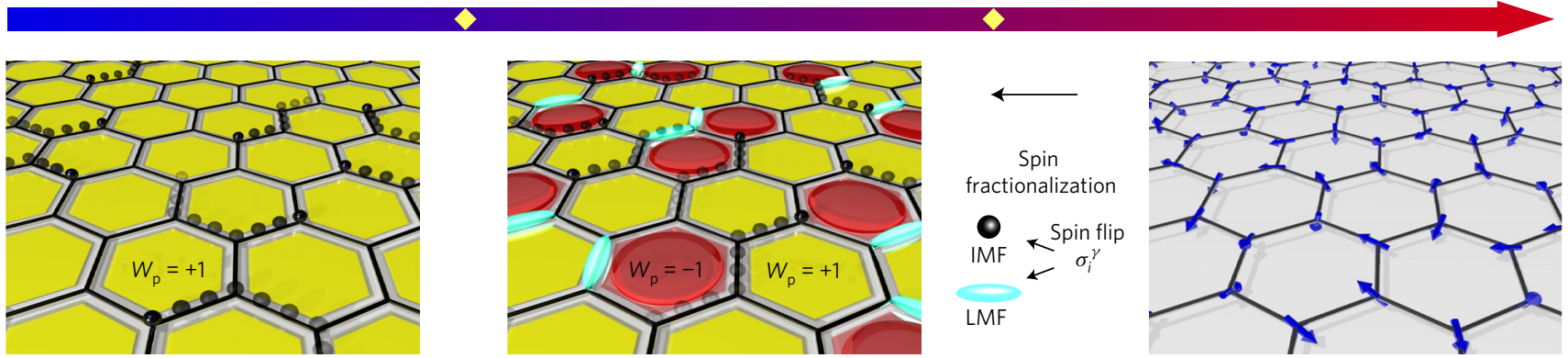

Figure 1 | Kitaev bonding geometry and cartoon of emergent Majorana fermions. a, Local $\mathrm{Ru}^{3+}\left(J_{\text {eff }}=1 / 2 ; 4 d^{5}\right)$ hexagon structure formed by the edge-shared $\mathrm{RuCl}_{6}$ octahedra, in the layered honeycomb material $\alpha-\mathrm{RuCl}_{3}$. Two $94^{\circ} \mathrm{Ru}-\mathrm{Cl}-\mathrm{Ru}$ superexchange paths lead to the Kitaev interactions $J_{\mathrm{K}}^{\gamma} \mathrm{S}_{i}^{\gamma} \mathrm{S}_{j}^{\gamma}$ between two magnetic spins on adjacent $i$ and $j$ sites, and the three different links denoted with $\gamma(=x, y, z)$ contribute isotropic $J_{K}$ in the rhombohedral crystal structure. The Pauli spin operators can be represented by $\sigma_{i}^{\gamma}=i b_{i}^{\gamma} c_{i}$ in terms of localized $\left(b_{i}^{\gamma}\right)$ and itinerant $\left(c_{j}\right)$ Majorana fermions in an extended Hilbert space. The plaquette operator $W_{p}$, a product of the bond operators $u_{i j}^{\gamma}=i b_{i}^{\gamma} b_{j}^{\gamma}$ around the hexagon, results in the $Z_{2}$ gauge fluxes of 0 (eigenvalue $\left.w_{p}=+1\right)$ and $\pi\left(w_{p}=-1\right)$ in the Kitaev lattice. $\mathbf{b}$, (Left) In very low-temperature $T<T_{L}$, the $Z_{2}$-fluxes are almost frozen in the quantum spin liquid ground state (yellow hexagon) and only low-energy itinerant Majorana fermions (black balls) are thermally activated. As temperature increases, the spin excitations are thermally fractionalized into itinerant and localized (cyan ovals) Majorana fermions. (Middle) In the intermediate temperature $T_{\mathrm{L}}<T<T_{\mathrm{H}}$ the $\pi$-fluxes (red hexagons) become populated by the thermal energy and the itinerant Majorana fermions on the vertices move in a coherent manner. As temperature crosses over $T_{H}$, the nearest-neighbour spin-spin correlation is diminished. (Right) Finally, in high temperature $T \gg T_{H}$, the system becomes a conventional paramagnet.

Finally, the system ends in a conventional paramagnetic phase well above $T_{\mathrm{H}}$.

Figure 2 displays the thermodynamic signatures in the magnetic susceptibility $\chi(T)$, magnetic specific heat $C_{\mathrm{M}}$ and entropy $S_{\mathrm{M}}$ for fractionalized spin excitations. The static $\chi(T)$ of $\alpha-\mathrm{RuCl}_{3}$ deviates from the Curie-Weiss curve below $140 \mathrm{~K}$, indicating the onset of short-range spin correlations (Fig. 2a). The anomalies in $\chi(T)$ and $C_{\mathrm{M}}$ at $T_{\mathrm{N}}=6.5 \mathrm{~K}$ represent the onset of zigzag-type AFM order (Fig. 2a,b). $C_{\mathrm{M}}$ is obtained by subtracting the lattice contribution from the total specific heat $\left(C_{\mathrm{P}}\right)$ as described in the Supplementary Information. Besides the sharp anomaly at $T_{\mathrm{N}}, C_{\mathrm{M}}$ exhibits two broad maxima, one near $T_{\mathrm{N}}$ and the other around $T_{\mathrm{H}} \approx 100 \mathrm{~K}$, although the low- $T$ maximum feature is obscured by the AFM anomaly. As predicted in theory ${ }^{12,13}$, the high- and low- $T$ structures can be ascribed to the thermal excitations of itinerant and localized Majorana fermions, respectively. It is worth noting that $C_{\mathrm{M}}$ follows a linear $T$-dependence in the intermediate range $T_{N}<T<T_{H}$, reflecting metallic-like behaviour of itinerant Majorana fermions (inset of Fig. 2b).

Rather firm evidence is provided by the two-stage release of the entropy gain $S_{\mathrm{M}}(T)=\int C_{\mathrm{M}} / T \mathrm{~d} T$ (Fig. $2 \mathrm{c}$ ). The obtained $S_{\mathrm{M}}$ at $T=200 \mathrm{~K}$ is $5.13 \mathrm{~J} \mathrm{~mol}^{-1} \mathrm{~K}^{-1}$, which corresponds to about $90 \%$ of the ideal value $R \ln 2$ ( $R$ : ideal gas constant) of the spin-1/2 system.
Upon cooling, nearly half of the entropy is released stepwise with the plateau-like behaviour at $0.46 R \ln 2$, signifying two maxima of $C_{\mathrm{M}}$. Indeed, $S_{\mathrm{M}}(T)$ above $T_{\mathrm{N}}$ agrees well with the simulated sum (red line) of two phenomenological Schottky-like functions with about an equal weight $\left(\rho_{\mathrm{H}}=0.92, \rho_{\mathrm{L}}=1.08, T_{\mathrm{H}} \simeq 101 \mathrm{~K}\right.$, and $\left.T_{\mathrm{L}} \simeq 22 \mathrm{~K}\right)$, which involve itinerant and localized Majorana fermions in the QMC simulation (see Supplementary Information). Considering the predicted temperature ratio $T_{\mathrm{L}} / T_{\mathrm{H}} \approx 0.03$ in the isotropic Kitaev model, $T_{\mathrm{L}}$ would be somewhat lower than $T_{\mathrm{N}}$ if the AFM order were absent. $S_{\mathrm{M}}$ involving AFM order below $T_{\mathrm{N}}$ was estimated to be $1.09 \mathrm{~J} \mathrm{~mol}^{-1} \mathrm{~K}^{-1}$, about $20 \%$ of the total entropy $R \ln 2$ ( $40 \%$ of $1 / 2 R \ln 2$ ) (ref. 27), indicating that the entropy held by the AFM order is partially released and roughly $3 / 5$ ths of the frozen $Z_{2}$-flux is maintained just above $T_{\mathrm{N}}$.

The microscopic and dynamic properties of the Majorana fermions can be visualized by the thermally fractionalized spin excitations obtained from the INS measurements. Figure $3 \mathrm{a}$ shows the neutron scattering function $\mathcal{S}_{\text {tot }}(\mathbf{Q}, \omega)$ as a function of momentum transfer $\mathbf{Q}$ and energy transfer $\omega$ measured at $T=10 \mathrm{~K}$ above $T_{\mathrm{N}}$ along the $\mathrm{X}-\mathrm{K}-\Gamma-\mathrm{M}-\mathrm{Y}$ direction. $\mathcal{S}_{\text {tot }}(\mathbf{Q}, \omega)$ at sufficiently low $T$ can be approximated as the magnetic scattering function $\mathcal{S}_{\text {mag }}(\mathbf{Q}, \omega)$ although weak phonon features are still observable as marked with black stars in the figure 


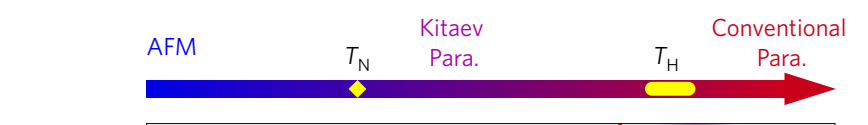

a

b

C

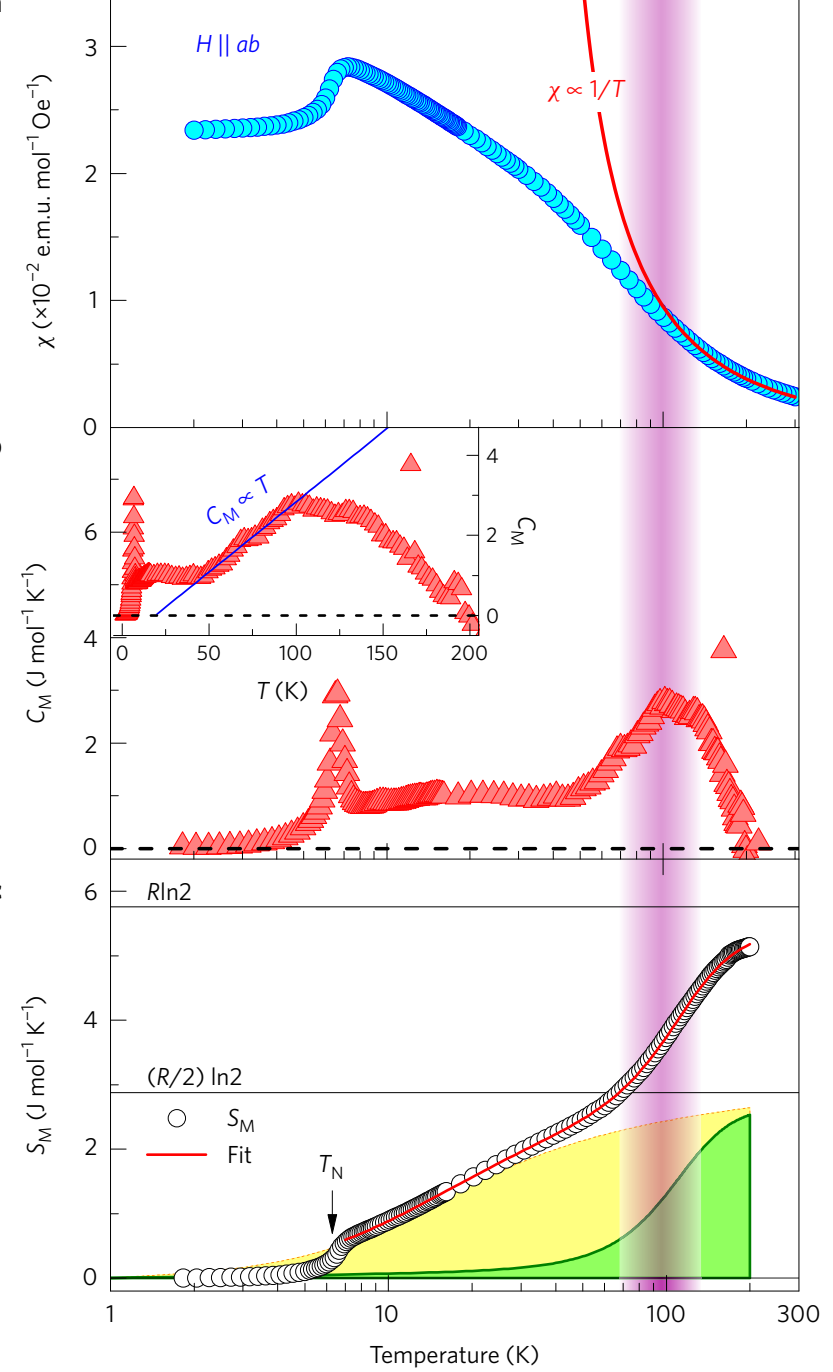

Figure 2 | Thermodynamic signatures of spin fractionalization.

a, Temperature-dependent static magnetic susceptibility of $\alpha-\mathrm{RuCl}_{3}$ plotted in a semi-log scale for $H \| a b$. The susceptibility deviates from the Curie-Weiss behaviour (solid red line) below $T=140 \mathrm{~K}$. The low-temperature kink at $T_{N}=6.5 \mathrm{~K}$ indicates a zigzag-type AFM order. b, Magnetic specific heat $C_{M}$ obtained by subtracting the lattice contribution in a semi-log scale (see Supplementary Information). Besides the AFM peak at $T_{\mathrm{N}}$, the broad bumps in $T_{\mathrm{N}} \lesssim T \lesssim 50 \mathrm{~K}$ and around $T=T_{H} \simeq 100 \mathrm{~K}$ (vertical bar) are associated with excitations of localized and itinerant Majorana fermions, respectively. $C_{M}$ exhibits a $T$-linear dependence in the intermediate temperature $50 \mathrm{~K} \lesssim T \lesssim T_{H}$, as shown in the inset, reflecting metal-like density of states of the itinerant Majorana fermions. The spike at $165 \mathrm{~K}$ is due to a structural phase transition. c, Magnetic entropy change, integrated $C_{M}$, in the temperature range $2 \mathrm{~K}<T<200 \mathrm{~K}$. The horizontal solid lines represent the expected total entropy change $R \ln 2$ and its half value $(R / 2) \ln 2$. The solid red line is a sum of two phenomenological function fits based on the theoretical simulation, indicating that the entropy release is decomposed into two fermionic components (yellow and green shadings), as described in the Supplementary Information.

(see Supplementary Information). $\mathcal{S}_{\text {tot }}(\mathbf{Q}, \omega)$ displays an hourglass shape spectrum centred at the $\Gamma$-point extending to about $20 \mathrm{meV}$ with strong low-energy excitations around the $\Gamma$-point and high-energy Y-shaped excitations. Similar features are reproduced in the simulated spectra of the isotropic Kitaev model with a FM Kitaev interaction $J_{\mathrm{K}}=-16.5 \mathrm{meV}$ by using the CDMFT + continuous-time QMC method ${ }^{14}$ (see Fig. $3 \mathrm{~b}$ ). It is worth noting that the spectral centre would move to the M-point for an $\mathrm{AFM} J_{\mathrm{K}}(>0)$ (ref. 14). The low-energy feature represents the quasielastic responses associated with the flux excitations, and the Y-shaped Q- $\omega$ dependence in the high-energy region reflects the dispersive itinerant Majorana fermions extending to $\omega \sim\left|J_{\mathrm{K}}\right|$ (refs 11,14). Both features are also clearly observable in the constantenergy cuts $\mathcal{S}_{\text {tot }}(\mathbf{Q})$, which also agree well with the theoretical calculations (Fig. 3c). According to the simulation, the excitation energy of the itinerant MF at the K- and M-points corresponds to Kitaev $J_{\mathrm{K}}$. $\mathcal{S}_{\text {tot }}(\mathbf{Q})$ data (Fig. 3d) are again compared with the simulated values (Fig. 3e) in 2D reciprocal space (Fig. 3f). The overall features are well reproduced by the simulations, except the hexagram-shaped Q-dependence of the low-energy $\mathcal{S}_{\text {tot }}(\mathbf{Q})(\omega \lesssim 6 \mathrm{meV})$, indicating that the key character of the Majorana fermions is rather robust. The hexagram-shaped $\mathbf{Q}$-dependence is considered to be induced by the second nearest-neighbour Kitaev interactions ${ }^{28}$ and/or symmetric anisotropy exchange interactions ${ }^{29,30}$ involving direct $\mathrm{Ru}-\mathrm{Ru}$ electron hopping, both of which are not considered in the pure Kitaev model. These interactions are weak, but become important at low energies and temperatures.

Figure $4 \mathrm{a}, \mathrm{b}$ presents the thermal evolution of the experimental and simulated $\mathcal{S}_{\operatorname{mag}}(\mathbf{Q}, \omega)$ (see Methods), respectively. At $T=16 \mathrm{~K}$, the hour-glass shape spectrum is maintained with minor reduction in the overall intensity. Upon heating up to $T_{\mathrm{H}} \sim 100 \mathrm{~K}$ (Kitaev paramagnetic phase), the low-energy intensity involving localized Majorana fermions is significantly reduced while the high-energy intensity from itinerant Majorana fermions is almost maintained, although the dichotomic feature becomes smeared with increasing thermal fluctuations. Further heating across $T_{\mathrm{H}}$ causes the highenergy intensity to begin to decrease considerably. Well above $T_{\mathrm{H}}(T=240 \mathrm{~K}), \mathcal{S}_{\mathrm{mag}}(\mathbf{Q}, \omega)$ exhibits only a featureless low background as in conventional paramagnets. The evolution of localized and itinerant Majorana fermions with temperature are visualized in the temperature-energy contour plots of $\mathcal{S}_{\text {mag }}$ around the $\Gamma$-point, as presented in Fig. 4c (experiment) and $4 \mathrm{~d}$ (simulation). The lowenergy excitations below $\omega \approx 4 \mathrm{meV}$ appear at $T \lesssim T_{\mathrm{H}}$ while the high-energy excitations extend out to $\omega \sim\left|J_{\mathrm{K}}\right|$. This is also evident from the $\mathcal{S}_{\text {mag }}(\Gamma, \omega)$ plots in Fig. $4 \mathrm{e}$, which are consistent with the simulations.

The quantitative agreement between the experiment and the simulation is also excellent in the INS intensities for the lowand high-energy excitations in an overall temperature range, as shown in Fig. 4f,g, presenting the temperature dependences of the corresponding integrations $\int \mathcal{S}_{\text {mag }}(\Gamma, \omega) \mathrm{d} \omega$. Meanwhile, one also notices that the experiment deviates somewhat from the simulation below $\sim 50 \mathrm{~K}$ only in the integration involving the lowenergy excitations (Fig. 4f). This is probably due to the presence of the additional perturbing magnetic interactions in the real system, whose influence might be apparent in the low-energy scale to be detrimental to the low-energy flux excitations at low temperature. Those perturbing interactions contribute the hexagramshaped $\mathbf{Q}$-dependence in the low-energy $\mathcal{S}_{\mathrm{mag}}(\mathbf{Q})$ (see Fig. 3d), which becomes isotropic above $\sim 50 \mathrm{~K}$, as expected in the Kitaev model (see Supplementary Information).

Tracing the magnetic entropy and evolution of the spin excitations as a function of temperature, energy, and momentum, we provide strong evidence for thermal fractionalization to Majorana fermions of spin excitations. $\alpha-\mathrm{RuCl}_{3}$ is well described in the ferromagnetic Kitaev model and is proximate to the Kitaev QSL. The key features of the thermal fractionalization predicted in the pure Kitaev model are reproduced well in the thermodynamic and spectroscopic results, although AFM order is developed below 


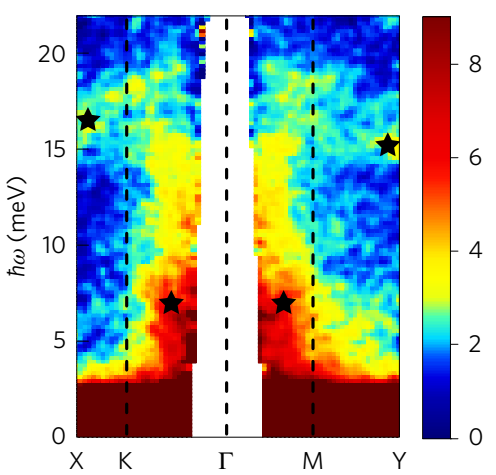

b

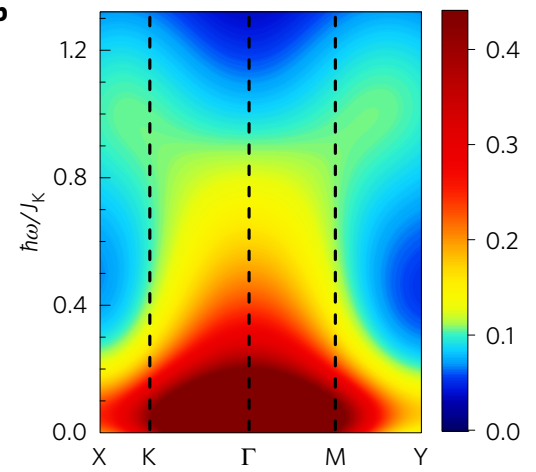

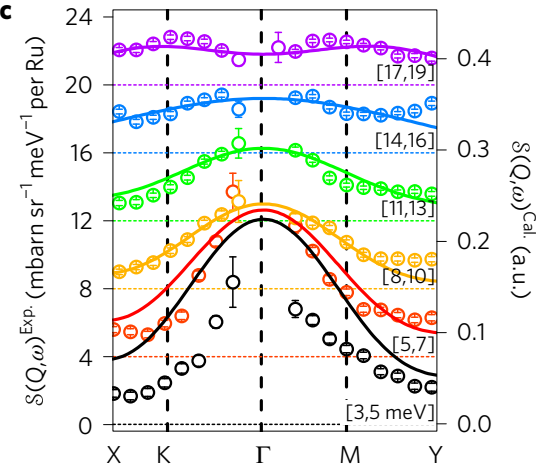
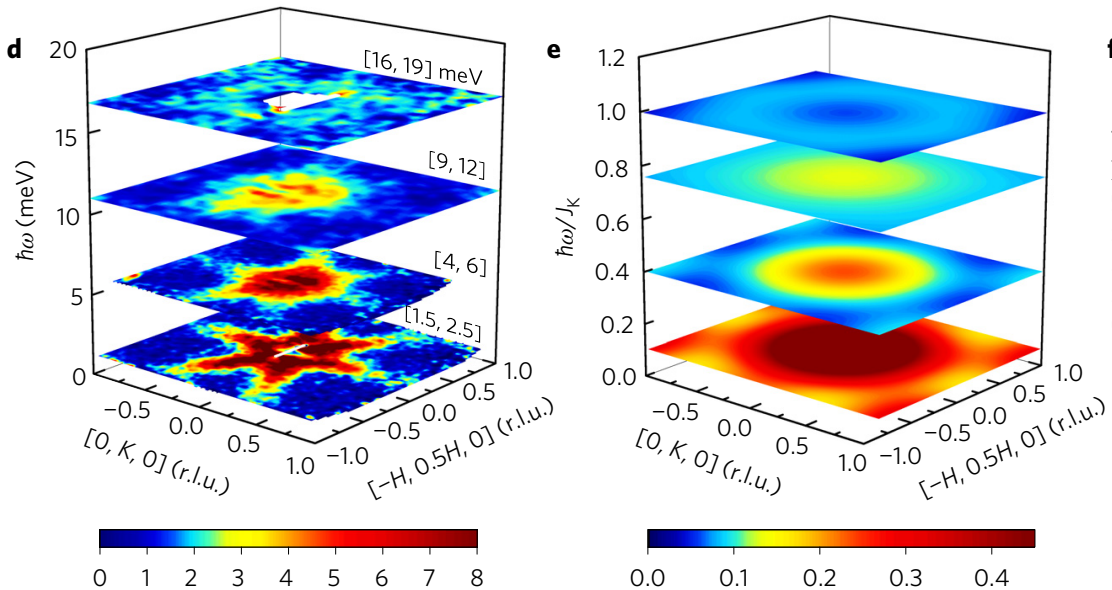

f

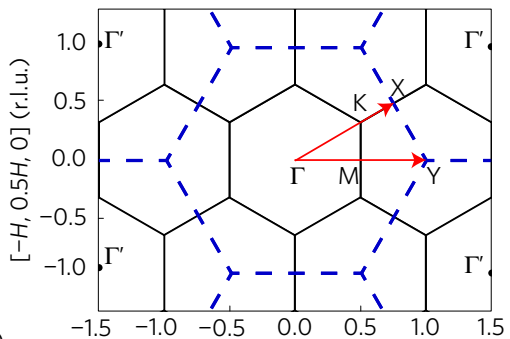

$[0, K, 0]$ (r.l.u.)

Figure 3 | Magnetic excitation spectra of $\alpha-\mathrm{RuCl}_{3}$ compared with the theoretical calculations on a ferromagnetic Kitaev model. a, Neutron scattering function $\mathcal{S}_{\text {tot }}(\mathbf{Q}, \omega)$ at $T=10 \mathrm{~K}$ along the high symmetric line $\mathrm{X}-\mathrm{K}-\Gamma-\mathrm{M}-\mathrm{Y}$ through Brillouin zone. The data were collected with an incoming neutron energy of $E_{\mathrm{i}}=31 \mathrm{meV}$ (MERLIN). The black stars mark phonons (see Supplementary Information). b, Calculated magnetic scattering function $\mathcal{S}_{\text {mag }}(\mathbf{Q}, \omega)$ for a ferromagnetic Kitaev model at $T=0.06\left|J_{K}\right|$. c, Constant-energy cuts integrated over the energy ranges $[3,5],[5,7],[8,10],[11,13],[14,16]$, and $[17,19]$ meV along the $\mathrm{X}-\mathrm{K}-\Gamma$ and $\Gamma-\mathrm{M}-\mathrm{Y}$ directions (left $\mathrm{y}$-axis). The dashed lines guide vertical offsets. The solid lines present the theoretical calculations of the pure Kitaev model (right $y$-axis). Error bars represent one standard deviation. d, Constant-energy cuts in the (hk)-plane integrated over the energy ranges $[1.5,2.5],[4,6]\left(L E T, E_{i}=10 \mathrm{meV}\right),[9,12]\left(\operatorname{LET}, E_{\mathrm{i}}=22 \mathrm{meV}\right)$, and $[16,19] \mathrm{meV}$ (MERLIN, $\left.E_{\mathrm{i}}=31 \mathrm{meV}\right)$. e, Constant-energy cuts of the theoretical $\mathcal{S}_{\text {mag }}(\mathbf{Q})$ in the Kitaev model for comparison. $\mathbf{f}$, The reciprocal honeycomb lattice in the $R \overline{3}$ space group. The $\mathrm{X}-\mathrm{K}-\Gamma$ and $\Gamma-\mathrm{M}-\mathrm{Y}$ directions are presented with the red arrows. The white regions in $\mathbf{a}, \mathbf{d}$ mark the lack of detector coverage. The colour bars in $\mathbf{a}, \mathbf{d}$ are represented in units of $\mathrm{mbarn} \mathrm{sr}^{-1} \mathrm{meV}^{-1}$ per $\mathrm{Ru}$. The calculations presented in $\mathbf{b}, \mathbf{e}$ are dimensionless, with the scale given by the colour bar.

$T_{\mathrm{N}}=6.5 \mathrm{~K}$ and additional perturbing magnetic interactions deteriorate QSL behaviour, especially in the low-energy scale. When the temperature is higher than the energy scale related to the perturbing magnetic interactions, the two distinct Majorana fermions predicted in the Kitaev honeycomb model are unveiled. This finding lays a cornerstone for an in-depth understanding of emergent Majorana quasiparticles in condensed matter, and also possibly for future implementation in quantum computations.

\section{Methods}

Methods, including statements of data availability and any associated accession codes and references, are available in the online version of this paper.

\section{Received 21 March 2017; accepted 16 August 2017;} published online 18 September 2017

\section{References}

1. Stormer, H. L., Tsui, D. C. \& Gossard, A. C. The fractional quantum Hall effect. Rev. Mod. Phys. 71, S298-S305 (1999).

2. Hasan, M. Z. \& Kane, C. L. Colloquium: topological insulators. Rev. Mod. Phys. 82, 3045-3067 (2010)

3. Xu, S. Y. et al. Discovery of a Weyl fermion semimetal and topological Fermi arcs. Science 349, 613-617 (2015).
4. Balents, L. Spin liquids in frustrated magnets. Nature 464, 199-208 (2010).

5. Kitaev, A. Anyons in an exactly solved model and beyond. Ann. Phys. 321, 2-111 (2006).

6. Anderson, $\mathrm{P}$. W. The resonating valence bond state in $\mathrm{La}_{2} \mathrm{CuO}_{4}$ and superconductivity. Science 235, 1196-1198 (1987).

7. Han, T.-H. et al. Fractionalized excitations in the spin-liquid state of a kagome-lattice antiferromagnet. Nature 492, 406-410 (2012).

8. Nayak, C., Simon, S. H., Stern, A., Freedman, M. \& Das Sarma, S. Non-Abelian anyons and topological quantum computation. Rev. Mod. Phys. 80, 1083-1159 (2008).

9. Elliott, S. R. \& Franz, M. Colloquium: Majorana fermions in nuclear, particle, and solid-state physics. Rev. Mod. Phys. 87, 137-163 (2015).

10. Baskaran, G., Mandal, S. \& Shankar, R. Exact results for spin dynamics and fractionalization in the Kitaev model. Phys. Rev. Lett. 98, 247201 (2007)

11. Knolle, J., Kovrizhin, D. L., Chalker, J. T. \& Moessner, R. Dynamics of a two-dimensional quantum spin liquid: signatures of emergent Majorana fermions and fluxes. Phys. Rev. Lett. 112, 207203 (2014).

12. Nasu, J., Udagawa, M. \& Motome, Y. Thermal fractionalization of quantum spins in a Kitaev model: temperature-linear specific heat and coherent transport of Majorana fermions. Phys. Rev. B 92, 115122 (2015).

13. Yamaji, Y. et al. Clues and criteria for designing a Kitaev spin liquid revealed by thermal and spin excitations of the honeycomb iridate $\mathrm{Na}_{2} \mathrm{IrO}_{3}$. Phys. Rev. $B$ 93, 174425 (2016)

14. Yoshitake, J., Nasu, J. \& Motome, Y. Fractional spin fluctuations as a precursor of quantum spin liquids: Majorana dynamical mean-field study for the Kitaev model. Phys. Rev. Lett. 117, 157203 (2016). 

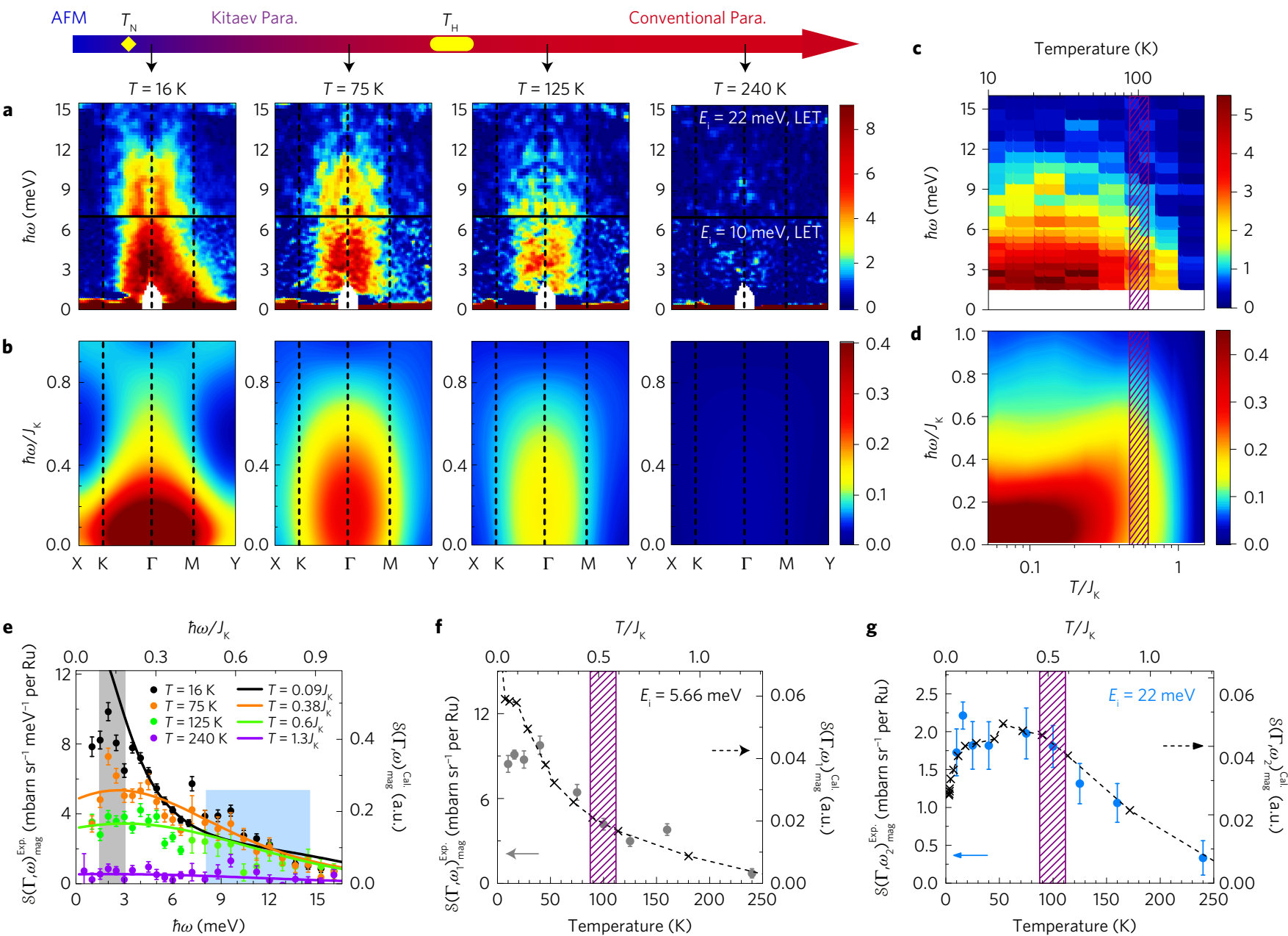

Figure 4 | Evolution of the two Majorana fermion excitations. a, Magnetic scattering function $\mathcal{S}_{\mathrm{mag}}(\mathbf{Q}, \omega)$ at $T=16,75,125$, and $240 \mathrm{~K}$. The two data sets with an incoming neutron energy of $E_{\mathrm{i}}=22 \mathrm{meV}$ (upper panel) and $10 \mathrm{meV}$ (down panel) are combined together. The white regions mark the lack of detector coverage. $\mathbf{b}$, Calculated $\mathcal{S}_{\operatorname{mag}}(\mathbf{Q}, \omega)$ at $T=0.09,0.375,0.69$, and $1.32\left|J_{K}\right|$ with $J_{K}=-16.5$ meV for comparison with the experimental data. $\mathbf{c}, \mathbf{d}$, Comparison of contour plot of the experimental $\mathcal{S}_{\text {mag }}(\Gamma, \omega)$ and the calculated $\mathcal{S}_{\mathrm{mag}}(\Gamma, \omega)$ in the temperature-energy plane. e, $\mathcal{S}_{\mathrm{mag}}(\Gamma, \omega)$ at $T=16 \mathrm{~K}$ (black circles), $75 \mathrm{~K}$ (yellow circles), $125 \mathrm{~K}$ (green circles), and $240 \mathrm{~K}$ (blue circles) as a function of energy. The calculated $\mathcal{S}_{\mathrm{mag}}(\Gamma, \omega)$ (the solid lines) are presented together for comparison. $\mathbf{f}, \mathbf{g}$, Temperature dependence of the integrated $\mathcal{S}_{\text {mag }}(\Gamma, \omega)$ over the energy range $\omega_{1}=[1.5,3]$ meV (grey circles) and $\omega_{2}=[8,14.5] \mathrm{meV}$ (blue circles). Both energy ranges are marked with grey and blue areas in e, respectively. The cross symbols represent the calculated results of the integrated $\mathcal{S}_{\text {mag }}(\Gamma, \omega)$, and the dashed lines represent the linear interpolations. The experimental $\mathcal{S}_{\text {mag }}(\Gamma, \omega)$ in $\mathbf{c}, \mathbf{e}-\mathbf{g}$ are obtained by integrating the $\mathcal{S}_{\text {mag }}(\mathbf{Q}, \omega)$ within the area $|H| \leq 0.12$ (reciprocal lattice unit) and $|K| \leq 0.2$ in the $H K$-plane. The areas of diagonal stripes in $\mathbf{c}, \mathbf{d}, \mathbf{f}, \mathbf{g}$ indicate the high- $T$ crossover at $T_{H}$. The colour bars in $\mathbf{a}, \mathbf{c}$ are represented in units of $\mathrm{mbarn} \mathrm{sr}^{-1} \mathrm{meV}^{-1} \mathrm{per} \mathrm{Ru}$. The calculations presented in $\mathbf{b}, \mathbf{d}$ are dimensionless, with the scale given by the colour bar. In $\mathbf{e}-\mathbf{g}$, measured and calculated $\mathcal{S}_{\text {mag }}$ refer to the left and right $y$-axes, respectively. Error bars represent one standard deviation.

15. Kim, B. J. et al. Novel $J_{\text {eff }}=1 / 2$ Mott state induced by relativistic spin-orbit coupling in $\mathrm{Sr}_{2} \mathrm{IrO}_{4}$. Phys. Rev. Lett. 101, 076402 (2008).

16. Jackeli, G. \& Khaliullin, G. Mott insulators in the strong spin-orbit coupling limit: from Heisenberg to a quantum compass and Kitaev models. Phys. Rev. Lett. 102, 017205 (2009).

17. Choi, S. K. et al. Spin waves and revised crystal structure of honeycomb iridate $\mathrm{Na}_{2} \mathrm{IrO}_{3}$. Phys. Rev. Lett. 108, 127204 (2012).

18. Ye, F., Chi, S., Cao, H. \& Chakoumakos, B. C. Direct evidence of a zigzag spin-chain structure in the honeycomb lattice: a neutron and $\mathrm{X}$-ray diffraction investigation of single-crystal $\mathrm{Na}_{2} \mathrm{IrO}_{3}$. Phys. Rev. B 85, 180403 (2012).

19. Plumb, K. W., Clancy, J. P., Sandilands, L. J. \& Shankar, V. V. $\alpha-\mathrm{RuCl}_{3}$ : a spin-orbit assisted Mott insulator on a honeycomb lattice. Phys. Rev. B 90, 041112 (R) (2014)

20. Koitzsch, A. et al. $J_{\text {eff }}$ description of the honeycomb Mott insulator $\alpha-\mathrm{RuCl}_{3}$. Phys. Rev. Lett. 117, 126403 (2016).

21. Sandilands, L. J., Tian, Y., Plumb, K. W., Kim, Y.-J. \& Burch, K. S. Scattering continuum and possible fractionalized excitations in $\alpha-\mathrm{RuCl}_{3}$. Phys. Rev. Lett. 114, 147201 (2015).

22. Banerjee, A. et al. Proximate Kitaev quantum spin liquid behaviour in a honeycomb magnet. Nat. Mater. 15, 733-740 (2016).
23. Johnson, R. D. et al. Monoclinic crystal structure of $\alpha-\mathrm{RuCl}_{3}$ and the zigzag antiferromagnetic ground state. Phys. Rev. B 92, 235119 (2015)

24. Cao, H. B. et al. Low-temperature crystal and magnetic structure of $\alpha-\mathrm{RuCl}_{3}$. Phys. Rev. B 93, 134423 (2016).

25. Winter, S. M., Li, Y., Jeschke, H. O. \& Valenti, R. Challenges in design of Kitaev materials: Magnetic interactions from competing energy scales. Phys. Rev. B 93, 214431 (2016).

26. Yadav, R. et al. Kitaev exchange and field-induced quantum spin-liquid states in honeycomb $\alpha-\mathrm{RuCl}_{3}$. Sci. Rep. 6, 37925 (2016).

27. Park, S. Y. et al. Emergence of the isotropic Kitaev honeycomb lattice with two-dimensional Ising universality in $\alpha-\mathrm{RuCl}_{3}$. Preprint at http://arxiv.org/abs/1609.05690v1 (2016).

28. Banerjee, A. et al. Neutron scattering in the proximate quantum spin liquid $\alpha-\mathrm{RuCl}_{3}$. Science 356, 1055-1059 (2017).

29. Catuneanu, A., Yamaji, Y., Wachtel, G., Kee, H.-Y. \& Kim, Y. B. Realizing quantum spin liquid phases in spin-orbit driven correlated materials. Preprint at http://arxiv.org/abs/1701.07837v1 (2017).

30. Ran, K. et al. Spin-wave excitations evidencing the Kitaev interaction in single crystalline $\alpha-\mathrm{RuCl}_{3}$. Phys. Rev. Lett. 118, 107203 (2017). 


\section{Acknowledgements}

This work is supported by the National Research Foundation (NRF) through the Ministry of Science, ICP \& Future Planning (MSIP) (NRF-2016K1A4A4A01922028). S.J. acknowledges support from the NRF grant (NRF-2017R1D1A1B03034432). S.-H.D. and K.-Y.C. are supported by the Korea Research Foundation (KRF) grant

(No. 2009-0076079) funded by the Korea government (MEST). J.Y., J.N. and Y.M. acknowledges Grant-in-Aid for Scientific Research under Grant No. JP15K13533, JP16K17747, and JP16H02206. Y.S.K. is supported from the NRF grant

(NRF-2015M2B2A9028507). K.K. acknowledges partial support from NRF grant (NRF-2016R1D1A1B02008461).

\section{Author contributions}

K.-Y.C. and S.J. conceived and designed the experiments. S.-H.D., Y.S.K. and K.-Y.C. synthesized single crystals. S.-H.D., S.-Y.P., T.-H.J. and K.-Y.C. measured and analysed the magnetic susceptibility and specific heat. S.-H.D., S.-Y.P. and S.J. performed inelastic neutron scattering measurements with the support from D.T.A. and D.J.V. S.-H.D., S.-Y.P and S.J. analysed neutron data. J.Y., J.N. and Y.M. carried out the CDMFT+CTQMC calculations. K.K. calculated magnetic form factors. S.-H.D., J.-H.P., K.-Y.C. and S.J. participated in writing of the manuscript. All authors discussed the results and commented on the manuscript. The project was led by S.J. and K.-Y.C. under the supervision of J.-H.P.

\section{Additional information}

Supplementary information is available in the online version of the paper. Reprints and permissions information is available online at www.nature.com/reprints. Publisher's note: Springer Nature remains neutral with regard to jurisdictional claims in published maps and institutional affiliations. Correspondence and requests for materials should be addressed to J.-H.P., K.-Y.C. or S.J.

\section{Competing financial interests}

The authors declare no competing financial interests. 


\section{Methods}

Crystal growth. High-quality single crystals of $\alpha-\mathrm{RuCl}_{3}$ and their isostructural counterpart $\mathrm{ScCl}_{3}$ were grown by a vacuum sublimation method. A commercial $\mathrm{RuCl}_{3}\left(\mathrm{ScCl}_{3}\right)$ powder (Alfa Aesar) was thoroughly ground, and dehydrated in a quartz ampoule for a day. The ampoule was sealed in vacuum and placed in a temperature gradient furnace. The temperature of the $\mathrm{RuCl}_{3}\left(\mathrm{ScCl}_{3}\right)$ powder is set at $1,080^{\circ} \mathrm{C}\left(900^{\circ} \mathrm{C}\right)$. After dwelling for $5 \mathrm{~h}$, the furnace is cooled to $650^{\circ} \mathrm{C}\left(600^{\circ} \mathrm{C}\right)$ at a rate of $-2^{\circ} \mathrm{C}$ per hour. We obtained $\alpha-\mathrm{RuCl}_{3}\left(\mathrm{ScCl}_{3}\right)$ crystals black coloured (transparent) with shiny surfaces. Electron-dispersive X-ray measurements confirmed the stoichiometry of the $\mathrm{Ru}(\mathrm{Sc}): \mathrm{Cl}=1: 3$ ratio for the crystals.

Magnetic susceptibility and specific heat measurement. Magnetic susceptibility measurements were performed using a commercial superconducting quantum interference device (SQUID) (Quantum Design, model: MPMS-5XL). A single domain crystal $\left(3 \times 3 \times 1 \mathrm{~mm}^{3}, 20 \mathrm{mg}\right)$ was chosen for the measurements under an external magnetic field parallel to the $a b$-plane. Specific heat $C_{\mathrm{p}}$ was measured by using a conventional calorimeter of the Quantum Design Physical Property Measurement System (model: PPMS DynaCool) in a temperature range of $T=1.8-300 \mathrm{~K}$. The magnetic specific heat $C_{\mathrm{M}}$ of $\alpha-\mathrm{RuCl}_{3}$ was determined by subtracting the lattice contribution, which is supposed to be equivalent to the specific heat of the isostructural non-magnetic $\mathrm{ScCl}_{3}$ with mass scaling (see Supplementary Information).

Inelastic neutron scattering. Inelastic neutron scattering data were collected by using the time-of-flight spectrometers MERLIN (high intensity) and LET (high resolution) at the ISIS Spallation Neutron Source, the Rutherford Appleton Laboratory in the United Kingdom. Total 46 pieces $(\sim 1.35 \mathrm{~g})$ of $\alpha-\mathrm{RuCl}_{3}$ single crystals for MERLIN, and 153 pieces $(\sim 5.1 \mathrm{~g})$ for LET were prepared, and co-aligned with crystallographic $c$-axis surface normal on aluminium plates, resulting in a mosaic within $3^{\circ}$ (Supplementary Fig. 1). The samples were mounted in a liquid helium cryostat for temperature control ranging from $1.5 \mathrm{~K}$ to $270 \mathrm{~K}$. Due to the highly two-dimensional structure of $\alpha-\mathrm{RuCl}_{3}$, magnetic correlations between honeycomb layers are extremely weak and insensitive. Therefore, crystals are aligned with the $c$-axis parallel to the incident neutron beam, so that the area detector measures the energy spectrum over the $2 \mathrm{D} q$-space of the $h k$-plane. To observe the intensity at the $\Gamma$-point (LET measurement), we rotated the crystal by 30 degrees to the incident beam direction, so that it filled the blank region of the beam mask.

Data were obtained with the incident neutron energy set to $E_{\mathrm{i}}=5.66,10,22$ (LET), and $31 \mathrm{meV}$ (MERLIN). With incoherent neutron scattering intensity measured from a vanadium standard sample, all data were normalized and converted to the value of the neutron scattering function $\mathcal{S}_{\text {tot }}(\mathbf{Q}, \omega)$, which is proportional to the differential neutron cross-section $\left(\mathrm{d}^{2} \sigma\right) /(\mathrm{d} \Omega \mathrm{d} E)$ and the ratio of the incident to the scattered neutron wavevector $k_{i} / k_{f}$ (ref. 31),

$$
\mathcal{S}_{\mathrm{tot}}(\mathbf{Q}, \omega) \sim \frac{k_{i}}{k_{f}} \frac{\mathrm{d}^{2} \sigma}{\mathrm{d} \Omega \mathrm{d} E}
$$

Since $\mathcal{S}_{\text {tot }}(\mathbf{Q}, \omega)$ contains both the nuclear and magnetic scattering contributions, the magnetic scattering function $\mathcal{S}_{\text {mag }}(\mathbf{Q}, \omega)_{T}$ at temperature $T$ in Fig. 4 is extracted from $\mathcal{S}_{\text {tot }}(\mathbf{Q}, \omega)_{T}$ after subtraction of the scaled $\mathcal{S}_{\text {tot }}(\mathbf{Q}, \omega)_{T_{0}=290 \mathrm{~K}}$ with the Bose factor correction $n(T) / n\left(T_{0}\right)=\left(1-\mathrm{e}^{-\hbar \omega / k_{\mathrm{B}} T_{0}}\right) /\left(1-\mathrm{e}^{-\hbar \omega / k_{\mathrm{B}} T}\right)$, which represents the approximate phonon contribution in the experiment.

$$
\mathcal{S}_{\text {mag }}(\mathbf{Q}, \omega)_{T} \approx \mathcal{S}_{\text {tot }}(\mathbf{Q}, \omega)_{T}-\frac{n(T)}{n\left(T_{0}\right)} \mathcal{S}_{\text {tot }}(\mathbf{Q}, \omega)_{T_{0}}
$$

All of the data processes, including Bose factor correction and projection of the scattering function along appropriate directions, were performed using the HORACE software, which is published by ISIS ${ }^{32}$.

Calculation of the magnetic scattering function. The calculation of $\mathcal{S}_{\operatorname{mag}}(\mathbf{Q}, \omega)_{T}$ is performed by using the CDMFT + continuous-time QMC method as described in ref. 15. The Bose factor correction of the equation (2) is also applied to the simulation results for quantitative comparison with the experimental results in Fig. 4. All calculated results include the magnetic form factor of the $\mathrm{Ru}^{3+}$ ion, which is obtained by using the density functional theory method considering solid-state effects in $\alpha$ - $\mathrm{RuCl}_{3}$, as described in the Supplementary Information.

Data availability. The data that support the plots within this paper and other findings of this study are available from the corresponding author upon reasonable request.

\section{References}

31. Xu, G., Xu, Z. \& Tranquada, J. M. Absolute cross-section normalization of magnetic neutron scattering data. Rev. Sci. Instrum. 84, 083906 (2013).

32. Ewings, R. A. et al. Horace: software for the analysis of data from single crystal spectroscopy experiments at time-of-flight neutron instruments. Nucl. Instrum. Methods Phys. Res. A 834, 132-142 (2016). 